\title{
A Prediction Model for Prediabetes Risk in Middle-Aged and Elderly Populations: A Prospective Cohort Study in China
}

\author{
Jiahua Wu $(\mathbb{D}$, Jiaqiang Zhou, Xueyao Yin, Yixin Chen, Xihua Lin, Zhiye Xu, and Hong Li (iD) \\ Department of Endocrinology, Sir Run Run Shaw Hospital, Zhejiang University School of Medicine, 3 East Qingchun Road, \\ Hangzhou 310016, China \\ Correspondence should be addressed to Hong Li; srrshnfm@zju.edu.cn
}

Received 24 July 2021; Accepted 29 September 2021; Published 11 November 2021

Academic Editor: Muhittin Yurekli

Copyright $\odot 2021$ Jiahua Wu et al. This is an open access article distributed under the Creative Commons Attribution License, which permits unrestricted use, distribution, and reproduction in any medium, provided the original work is properly cited.

Background. To investigate indicators for prediabetes risk and construct a prediction model for prediabetes incidences in China. Methods. In this study, 551 adults aged 40-70 years had normal glucose tolerance (NGT) and normal hemoglobin A1c (HbA1c) levels at baseline. Baseline data including demographic information, anthropometric measurements, and metabolic profile measurements were collected. The associations between possible indicators and prediabetes were assessed by the Cox proportional-hazards model. The predictive values were evaluated by the area under the receiver operating characteristic (ROC) curve (AUC). Results. During an average of 3.35 years of follow-up, the incidence of prediabetes was found to be $19.96 \%(n=110)$. In the univariate analyses, fasting plasma glucose (FPG), fasting serum insulin (FINS), $2 \mathrm{~h}$ plasma glucose (2hPG), HbA1c, serum uric acid (SUA), waist circumference (WC), smoking, and family history of diabetes (FHD) were found to be significantly correlated with prediabetes. In the multivariable analyses, WC (hazard ratio (HR): 1.032; 95\% confidence interval (CI): $1.010,1.053$; $p=0.003$ ), FHD (HR: 1.824; 95\% CI: 1.250, 2.661; $p=0.002$ ), HbAlc (HR: 1.825; 95\% CI: 1.227, 2.714; $p=0.003$ ), and FPG (HR: 2.284; 95\% CI: 1.556, 3.352; $p<0.001)$ were found to be independent risk factors for prediabetes. A model that encompassed WC, FHD, HbA1c, and FPG for predicting prediabetes exhibited the largest discriminative ability (AUC: 0.702). Conclusions. WC, FHD, HbA1c, and FPG are independently correlated with the risk of prediabetes. Furthermore, the combination of these predictors enhances the predictive accuracy of prediabetes.

\section{Introduction}

Diabetes has evolved as a global health challenge. It is correlated with a high mortality rate, increased health risks, medical costs, and a poor quality of life. Prediabetes refers to the transition from normal glucose metabolism to diabetes, consisting of impaired fasting glucose (IFG) and impaired glucose tolerance (IGT) [1]. Prediabetic adults do not show any signs or symptoms of diabetes; therefore, many people are unaware that they are living with prediabetes [2]. Prediabetic individuals have an increased risk for diabetes, obesity, hypertension, dyslipidemia, cardiovascular disease, cancer, and dementia [3, 4]. Prediabetes prevention and control reduces the chances for progression to diabetes and its related complications.
In 2017, the World Health Organization documented that the global prevalence of diabetes and prediabetes has been on the rise [5]. Globally, China has the highest number of prediabetes patients, with a prevalence of $35.2 \%$ in 2017 . This corresponds to approximately 357 million people [6]. A Chinese study involving adults with IGT reported that the cumulative incidence of type 2 diabetes in 6 years was $67.7 \%$ [7]. Therefore, it is important to identify indicators that efficiently predict prediabetes development. This will help monitor patients and improve intervention.

Diabetes pathogenesis involves interactions between genes and the environment. Factors influencing prediabetes include age [8,9], educational attainment [9], marital status [9], hypertension [10], dyslipidemia [11], gestational diabetes, body mass index (BMI) $[9,12]$, WC [12], FHD [13], 
diet patterns [14], and 1-hour plasma glucose levels [15]. In Japan, a prognostic model based on six variables was built to predict the incidences of type 2 diabetes mellitus and prediabetes in a healthy population [16]. The AUC of the predictive model was found to be 0.87 . Moreover, a model for screening prediabetes in Indonesian adults (AUC = 0.623), which includes age, sex, education level, FHD, smoking, physical activity, BMI, and hypertension, has been developed and verified [17]. However, a prediction model for predicting prediabetes incidences in China has not been constructed. Therefore, we aimed at investigating the potential indicators and to construct a prediction model for prediabetes incidence among middle-aged and elderly populations in urban areas of Hangzhou, China.

\section{Methods}

2.1. Study Participants. Participants enrolled in this cohort study were from the Caihe community of Jianggan District, Hangzhou. Participants, aged between 40 and 70 years, were recruited by community workers. Recruitment was done between January and March 2010. Follow-up was done in 2011, 2013, and 2015. The exclusion criteria were as follows: (i) those using glucocorticoids; (ii) those with cirrhosis and ascites; (iii) hyperthyroidism or hypothyroidism; (iv) cancer; (v) severe disabilities or mental diseases; (vi) pregnant and lactating women; (vii) participants with diabetes or prediabetes at baseline; (viii) those with incomplete information; (ix) lost at follow-up; and $(\mathrm{x})$ new-onset diabetes before the diagnosis of prediabetes at follow-up. For all participants, there was at least one visit between 2011 and 2015. The study was approved by the ethics committee of Sir Run Run Shaw Hospital. Informed consent was provided by all participants.

2.2. Demographic Characteristics. Age, gender, education levels, nature of occupation, cigarette smoking habits, alcohol drinking habits, dietary patterns, regular exercise, and FHD data were obtained by a questionnaire. Participants were grouped based on cigarette smoking habits, that is, smoker, exsmoker, and nonsmoker. Moreover, they were categorized based on alcohol drinking habits, that is, drinker, exdrinker, and nondrinker. Education levels were categorized as middle school or above and below middle school. The nature of occupation before retirement was defined as manual work, physical and mental work, and mental work. Dietary patterns were divided into main meat dishes, balanced meat and vegetables, and main vegetable dishes. The FHD was defined as the presence of diabetes among first-degree relatives. Regular exercise was more than 1 day per week.

2.3. Assessment of Anthropometric Measurements and Metabolic Profiles. The measurement of WC, blood pressure, BMI, FPG, FINS, SUA, high-density lipoprotein cholesterol (HDL-C), triglyceride (TG) levels, and 2hPG levels was based on the previous article [18].
2.4. Definition. The definition of hypertension was systolic blood pressure (SBP) $\geq 140 \mathrm{mmHg}$ and/or diastolic blood pressure (DBP) $\geq 90 \mathrm{mmHg}$ and/or diagnosed as hypertension by a physician previously. The definition of diabetes was FPG $\geq 7.0 \mathrm{mmol} / \mathrm{L}, 2 \mathrm{hPG} \geq 11.1 \mathrm{mmol} / \mathrm{L}$, or previously diagnosed as diabetes. The definition of prediabetes was $7.0 \mathrm{mmol} /$ $\mathrm{L}>\mathrm{FPG} \geq 6.1 \mathrm{mmol} / \mathrm{L}, \quad 11.1 \mathrm{mmol} / \mathrm{L}>2 \mathrm{hPG} \geq 7.8 \mathrm{mmol} / \mathrm{L}$, or previously diagnosed as prediabetes.

2.5. Statistical Analysis. All analyses were performed with $\mathrm{R}$ 3.5.0 and SPSS 26.0. Continuous variables were shown as means \pm standard deviations, medians (interquartile ranges), or frequencies and percentages. Continuous variables with a skewed distribution were transformed by natural logarithm transformation before analysis. Comparison between 2 groups was conducted by independent-samples $t$-test for continuous variables, while $\chi^{2}$ tests were used for categorical variables. The multivariable Cox model was performed to estimate the correlations between indicators and prediabetes incidences. Clinically relevant baseline variables or variables with $p<0.2$ upon univariate analysis were entered into the multivariate analysis. Variables were entered into the multivariate Cox proportional-hazards model one by one. They were kept in the final models, which were added to this model, changing the matched hazard ratio by at least 10 percent or if the $p$ value by itself made sense. The variance inflation factor of the variables included in the model was examined to address collinearity. No evidence of collinearity was noted in the model, given the variance inflation factor of $<5$. The ROC was applied to compare the predictive accuracy of various models. Differences between AUC were determined using DeLong's test. Additionally, integrated discrimination improvement (IDI), net reclassification index (NRI), and Akaike's information criterion (AIC) were calculated to evaluate the predictive values of different models. $p \leq 0.05$ was considered statistically significant.

\section{Results}

3.1. Basic Characteristics of the Study Participants. A total of 1,030 participants were initially enrolled from January to March 2010. Among them, 223 had prediabetes or type 2 diabetes at baseline, 243 were lost at follow-up, while 5 participants had incomplete data. Eight participants were excluded because they were diagnosed as type 2 diabetes at follow-up. Finally, 551 eligible participants were enrolled (Figure 1). During an average of 3.35 years of follow-up, 110 of the 551 participants without dysglycemia at baseline developed prediabetes (incidence: 19.96\%). Demographic, clinical, and biological characteristics of 551 participants are shown in Table 1. Average age at baseline was $53.23 \pm 6.62$ years in the NGT group and $54.42 \pm 7.02$ years in the prediabetes group. Participants with prediabetes outcomes exhibited significantly elevated WC, BMI, FPG, 2hPG, HbA1c, FINS, and SUA levels as well as significantly suppressed HDL-C level at baseline. Moreover, at baseline, proportion of male, prevalence of hypertension, smoking, 


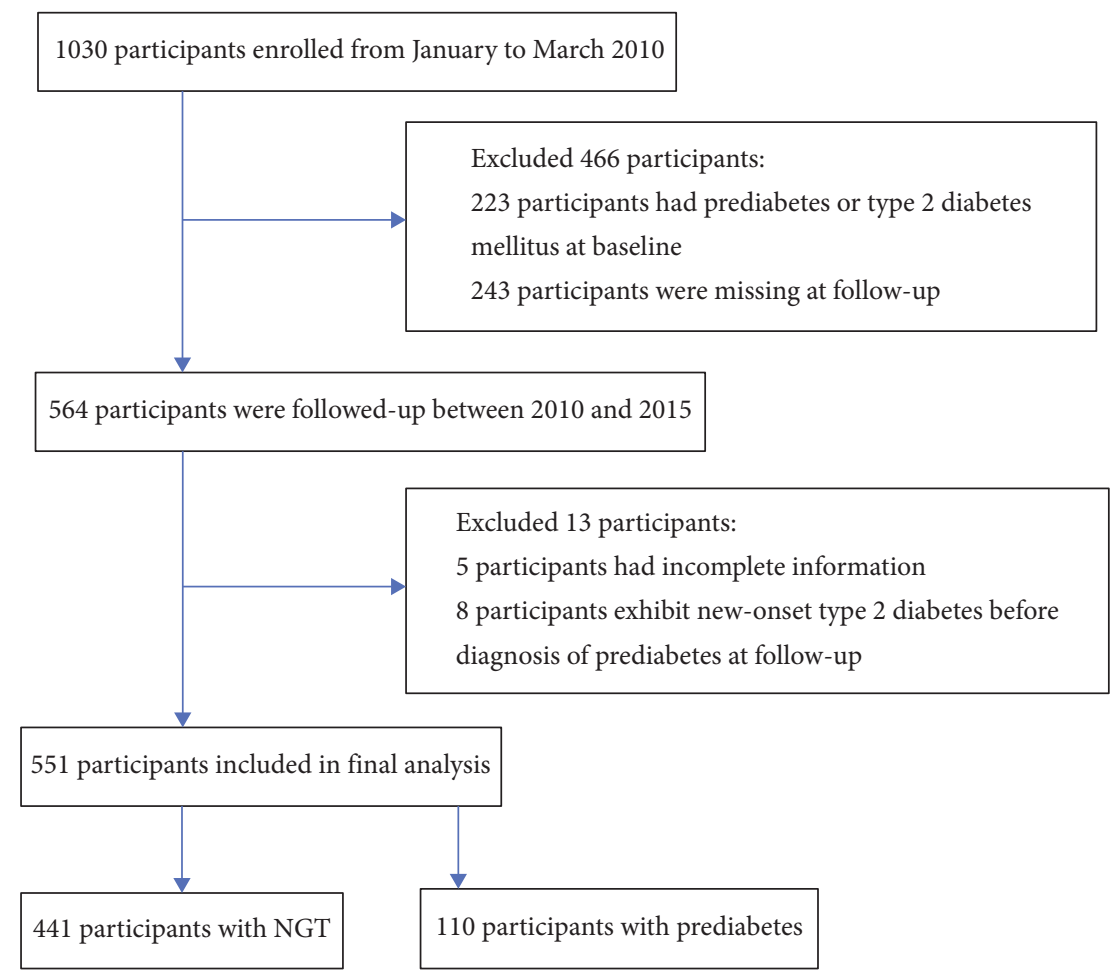

FIgURE 1: Schematic presentation for participant selection.

and FHD were significantly higher in participants with prediabetes. At baseline, there were no significant differences in age, TG, alcohol drinking, dietary patterns, nature of occupation, education level, and regular exercise.

\subsection{Associations between Possible Indicators and the Risk of} Prediabetes. In the univariate analyses (Additional file 1: Table S1), FPG, FINS, 2hPG, HbA1c, SUA, WC, smoking, and FHD were found to be significantly correlated with prediabetes. The FPG was the strongest predictor for prediabetes. In the multivariate model, 16 variables (age, gender, hypertension, smoking, drinking, FHD, regular exercise, WC, BMI, FPG, FINS, 2hPG, HbA1c, SUA, HDL$\mathrm{C}$, and TG) were selected into the model for screening. The final prognostic models are shown in Table 2. Given the relationship between $\mathrm{WC}$ and glucose metabolism, WC was set as the base model (model 1), which was easy to be clinically obtained. Model 2 additionally included FHD. The variables in model 2 plus HbAlc were selected for model 3. Model 4 additionally included FPG. Multivariate Cox proportional-hazard analyses showed that WC (HR: 1.032; 95\% CI: 1.010, 1.053; $p=0.003$ ), FHD (HR: 1.824; 95\% CI: $1.250,2.661 ; p=0.002$ ), HbA1c (HR: $1.825 ; 95 \%$ CI: 1.227 , $2.714 ; p=0.003$ ), and FPG (HR: 2.284 ; 95\% CI: 1.556, 3.352; $p<0.001)$ were independent risk factors of prediabetes.

3.3. Predictive Values of FPG, HbA1c, WC, and FHD for Prediabetes. The predictive accuracy of the resultant models for prediabetes was evaluated by ROC curve analysis (Figure 2). The established independent risk factors for prediabetes were used to construct the predictive model. The AUC was 0.637 when WC was used alone (model 1 ). The addition of FHD (model 2) in model 1 did not significantly increase the AUC (model 2 versus model 1: DeLong's test, $p=0.7901)$. However, a significant increase in AUC was observed when HbAlc and FPG were further added (model 3 versus model 2: DeLong's test, $p=0.0205$; model 4 versus model 3: DeLong's test, $p=0.0013$ ), with AUC eventually increasing to 0.702 (model 4). Discriminative abilities of the resultant models were confirmed using the NRI, IDI, and AIC measures (Table 3). Model 4 exhibited the smallest AIC measure and significantly enhanced risk reclassification and discrimination (NRI, 30.4\%; 95\% CI: 0-38.9\%; IDI, 10.4\%; 95\% CI: 1.7-21.5\%) when compared with model 1.

\section{Discussion}

In this study, we found that FPG, HbAlc, WC, and FHD are independently associated with the development of prediabetes among middle-aged and elderly adults in China. Moreover, the potential of the developed prognostic model for screening the individual risk of developing prediabetes was high.

People with visceral obesity are more likely to develop prediabetes [19]. Glucose utilization in the peripheral tissues and the liver is affected by changes in tissue proportions and increase of free fatty acids. This causes gluconeogenesis, which results in insulin resistance, thereby increasing the risk of prediabetes [20]. WC is a simple and commonly used marker for visceral obesity and is correlated with a higher risk of prediabetes among Chinese and Iranian adults [12]. Our study confirmed this finding. The WC was independently correlated with a 1.032-fold (95\% CI: $1.010-1.053$ ) 
TABLE 1: Basic characteristics of study participants categorized by glucose metabolism.

\begin{tabular}{|c|c|c|c|}
\hline Characteristic & NGT $(n=441)$ & Prediabetes $(n=110)$ & $p$ value \\
\hline Age, years & $53.23 \pm 6.62$ & $54.42 \pm 7.02$ & 0.093 \\
\hline Gender, men (\%) & $149(33.80)$ & $52(47.30)$ & 0.009 \\
\hline BMI, $\mathrm{kg} / \mathrm{m}^{2}$ & $23.01 \pm 2.67$ & $23.77 \pm 2.73$ & 0.007 \\
\hline $\mathrm{WC}, \mathrm{cm}$ & $76.47 \pm 8.40$ & $80.56 \pm 8.85$ & $<0.001$ \\
\hline $\mathrm{FPG}, \mathrm{mmol} / \mathrm{L}$ & $4.70 \pm 0.46$ & $4.98 \pm 0.58$ & $<0.001$ \\
\hline FINS, uIU/mL & $5.73 \pm 4.31$ & $7.54 \pm 6.41$ & $<0.001$ \\
\hline $2 \mathrm{hPG}, \mathrm{mmol} / \mathrm{L}$ & $5.03 \pm 1.14$ & $5.52 \pm 1.36$ & $<0.001$ \\
\hline $\mathrm{HbAlc}, \mathrm{mmol} / \mathrm{mol}$ & $36.32 \pm 4.57$ & $38.45 \pm 4.57$ & $<0.001$ \\
\hline HbAlc (\%) & $5.47 \pm 0.42$ & $5.67 \pm 0.42$ & $<0.001$ \\
\hline $\mathrm{SUA}, \mu \mathrm{mol} / \mathrm{L}$ & $272.77 \pm 84.21$ & $299.83 \pm 81.56$ & 0.003 \\
\hline${ }^{\mathrm{a}} \mathrm{TG}, \mathrm{mmol} / \mathrm{L}$ & $1.23(0.89-1.66)$ & $1.44(0.90-1.93)$ & 0.098 \\
\hline HDL-C, mmol/L & $1.50 \pm 0.36$ & $1.41 \pm 0.37$ & 0.015 \\
\hline Hypertension, $n(\%)$ & $94(21.30)$ & $34(30.90)$ & 0.033 \\
\hline Family history of diabetes, $n(\%)$ & $108(24.50)$ & $48(43.60)$ & $<0.001$ \\
\hline Regular exercise, $n(\%)$ & $271(61.50)$ & $64(58.20)$ & 0.530 \\
\hline Smoking, $n(\%)$ & & & 0.003 \\
\hline Current smokers & $92(20.90)$ & $26(23.60)$ & \\
\hline Former smokers & $17(3.90)$ & $13(11.80)$ & \\
\hline Never smokers & $332(75.30)$ & $71(64.50)$ & \\
\hline Alcohol drinking, $n(\%)$ & & & 0.195 \\
\hline Current drinkers & $85(19.30)$ & $26(23.60)$ & \\
\hline Former drinkers & $82(18.60)$ & $26(23.60)$ & \\
\hline Never drinkers & $274(62.10)$ & $58(52.70)$ & \\
\hline Dietary patterns, $n(\%)$ & & & 0.789 \\
\hline Main meat dishes & $30(6.80)$ & $8(7.30)$ & \\
\hline Balanced meat and vegetables & $319(72.30)$ & $76(69.10)$ & \\
\hline Main vegetable dishes & $92(20.90)$ & $26(23.60)$ & \\
\hline Nature of occupation, $n(\%)$ & & & 0.240 \\
\hline Manual work & $111(25.20)$ & $23(20.90)$ & \\
\hline Physical and mental work & $183(41.50)$ & $41(37.30)$ & \\
\hline Mental work & $147(33.30)$ & $46(41.80)$ & \\
\hline Education level, $n(\%)$ & & & 0.914 \\
\hline Middle school or below & $211(47.80)$ & $52(47.30)$ & \\
\hline High school or above & $230(52.20)$ & $58(52.70)$ & \\
\hline
\end{tabular}

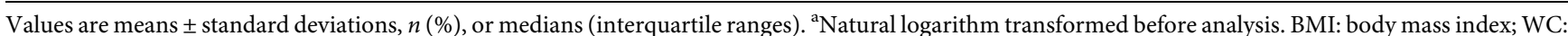
waist circumference; FPG: fasting plasma glucose; FINS: fasting serum insulin; HbAlc: hemoglobin Alc; HDL-C: high-density lipoprotein cholesterol; NGT: normal glucose tolerance; SUA: serum uric acid; TG: triglycerides; $2 \mathrm{hPG}$ : $2 \mathrm{~h}$ plasma glucose.

TABLE 2: Multivariable Cox proportional-hazards regression models for predicting prediabetes.

\begin{tabular}{|c|c|c|c|c|c|c|c|c|}
\hline \multirow{2}{*}{ Variable } & \multicolumn{2}{|l|}{ Model 1} & \multicolumn{2}{|l|}{ Model 2} & \multicolumn{2}{|l|}{ Model 3} & \multicolumn{2}{|l|}{ Model 4} \\
\hline & HR (95\% CI) & $p$ value & $\mathrm{HR}(95 \% \mathrm{CI})$ & $p$ value & HR $(95 \% \mathrm{CI})$ & $p$ value & $\operatorname{HR}(95 \% \mathrm{CI})$ & $p$ value \\
\hline $\mathrm{WC}$ & $1.039(1.018,1.061)$ & $<0.001$ & $039(1.018,1.060)$ & $<0.001$ & $1.039(1.018,1.061)$ & $<0.001$ & $1.032(1.010,1.053)$ & 0.003 \\
\hline FHD & - & - & $924(1.320,2.805)$ & 0.001 & $1.844(1.262,2$ & 0 . & $1.824(1.250,2.661)$ & 0.002 \\
\hline HbAlc & - & - & - & - & $2.028(1.382,2.976)$ & $<0.001$ & $1.825(1.227,2.714)$ & 0.003 \\
\hline FPG & - & - & - & - & - & - & $2.284(1.556,3.352)$ & $<0.001$ \\
\hline
\end{tabular}

Model 1: incorporating WC; model 2: variables in model 1 and FHD; model 3: variables in model 2 and HbAlc; model 4: variables in model 3 and FPG. CI, confidence intervals; FHD, family history of diabetes; FPG, fasting plasma glucose; HbA1c, hemoglobin A1c; HR, hazard ratios; WC, waist circumference.

increased risk of prediabetes. Despite the power of WC for predicting prediabetes, its predictive value was not strong enough as an independent predictor in the clinical setup. Other risk factors should be considered to improve its ability as a predictive tool for prediabetes.

FHD is a predictor for prediabetes development. A multicenter study found that FHD was significantly correlated with prediabetes development [21]. This relationship remained significant when adjusted for gender, age, and BMI. Moreover, FHD in a first-degree relative was associated with IFG among children and adolescents [22]. In this study, FHD was independently correlated with a 1.824 -fold $(95 \%$ CI: 1.250-2.661) increased risk of prediabetes. The results are in line with the reported familial clustering risk and may be related to genetics and family environment [23].

Besides WC and FHD, FPG was correlated with a higher risk for prediabetes in our study. FPG was independently correlated with a 2.284 -fold (95\% CI: $1.556-3.352)$ increased risk of prediabetes. This outcome has been confirmed in other studies. An increase in FPG within the normal range is 

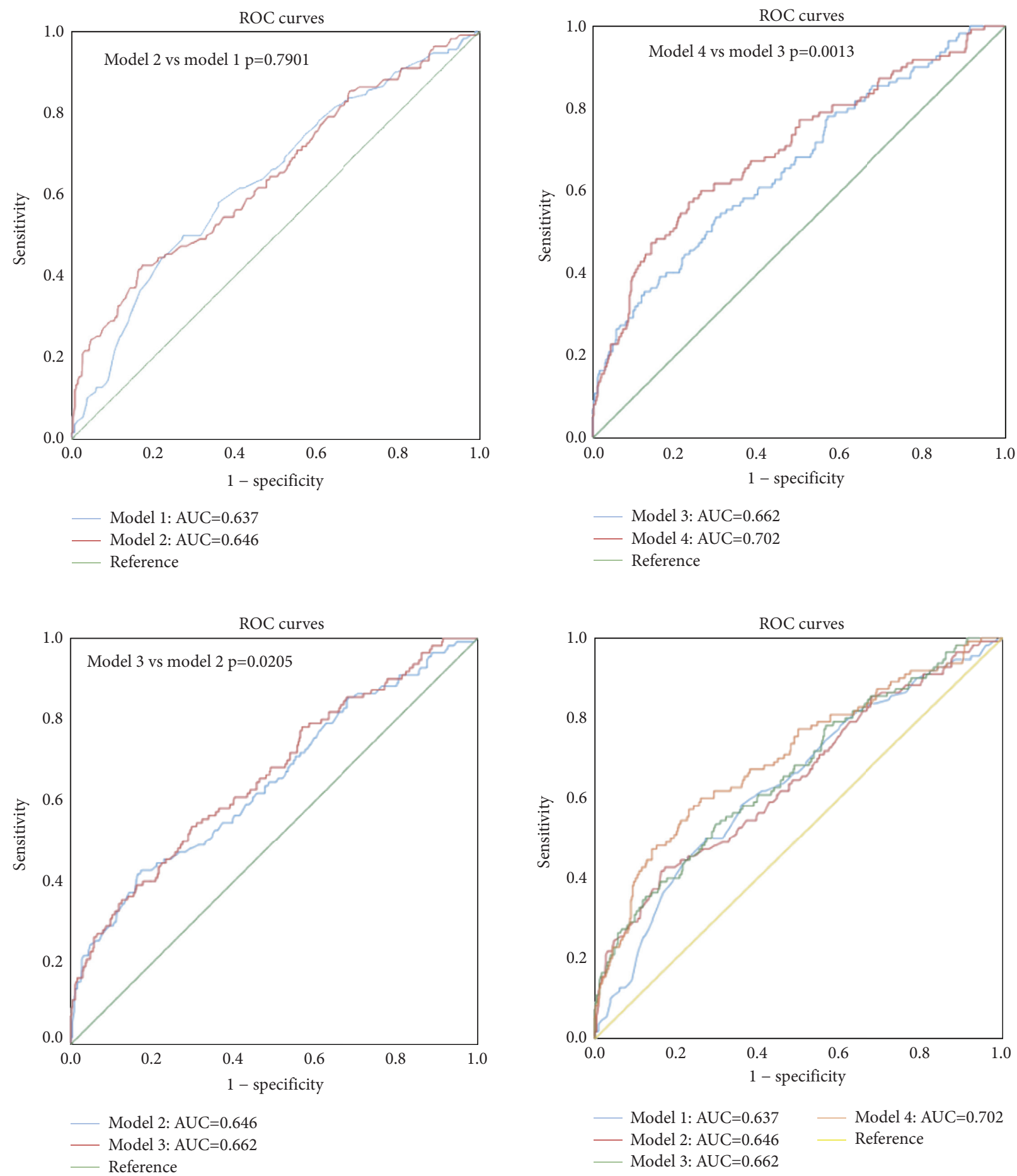

FIGURE 2: Area under the receiver operating characteristic curves for predicting prediabetes. Model 1: incorporating WC. Model 2: variables in model 1 and FHD. Model 3: variables in model 2 and HbA1c. Model 4: variables in model 3 and FPG. FHD: family history of diabetes; FPG: fasting plasma glucose; HbAlc: hemoglobin A1c; WC: waist circumference.

correlated with increased incidences of IGT and IFG [24]. Elevated FPG (within the normal range) is a predictor for prediabetes and diabetes development during youth, middle, and old ages [25]. These studies confirm that an increase in FPG is potentially useful in identifying healthy people who are at risk of developing prediabetes. Furthermore, we found that $\mathrm{HbAlc}$ is correlated with a higher risk for prediabetes.
This is the first work to be performed in China to determine whether HbAlc is beneficial in risk prediction of prediabetes. Interestingly, $\mathrm{HbAlc}$ was independently correlated with a 1.825 -fold (95\% CI: $1.227-2.714)$ increased risk of prediabetes.

Most of the domestic studies have used a single index to predict prediabetes. In a 2020 study, it was shown that the 
TABLE 3: Performance of models in prediabetes prediction.

\begin{tabular}{|c|c|c|c|c|c|}
\hline Model & AIC & NRI $(95 \%$ CI $)$ & $p$ value & IDI $(95 \% \mathrm{CI})$ & $p$ value \\
\hline Model 1 & 1275.641 & - & - & - & - \\
\hline Model 2 & 1266.549 & $9.1 \%(-7.3,22.8)$ & 0.348 & $1.4 \%(-0.7,5.7)$ & 0.279 \\
\hline Model 3 & 1257.077 & $23.5 \%(-1.2,31.3)$ & 0.139 & $3.4 \%(0,11.8)$ & 0.04 \\
\hline Model 4 & 1241.303 & $30.4 \%(0,38.9)$ & 0.04 & $10.4 \%(1.7,21.5)$ & $<0.01$ \\
\hline
\end{tabular}

Model 1: incorporating WC; model 2: variables in model 1 and FHD; model 3: variables in model 2 and HbA1c; model 4: variables in model 3 and FPG. AIC: Akaike's information criterion; CI: confidence interval; FHD: family history of diabetes; FPG: fasting plasma glucose; HbA1c: hemoglobin Alc; HR: hazard ratio; IDI: integrated discrimination improvement; NRI: net reclassification index; WC: waist circumference.

triglyceride-glucose index (TyG index) was considered as a potential predictor for identifying high-risk individuals with prediabetes. The AUC for the TyG index in predicting prediabetes was found to be 0.60 [11]. The Chinese visceral adiposity index (CVAI) is considered as a better indicator for predicting prediabetes than WC in Chinese adults. The AUC for the CVAI was found to be 0.64 in women and 0.57 in men [12]. Visceral adiposity index (VAI), waist-height ratio (WHtR), and WC were considered as independent predictors for prediabetes in women over 40 years. The AUC of VAI, WHtR, and WC for predicting prediabetes was 0.625 , 0.602 , and 0.598 , respectively [26]. All the aforementioned predictors were not independently strong enough to predict prediabetes in the clinical setup $(A U C<0.70)$. A combination of different predictors enhances the predictive accuracy for prediabetes. In this study, multivariate Cox proportional-hazard analyses revealed that WC, FHD, HbAlc, and FPG were independent risk factors of prediabetes. A combination of FPG, HbAlc, WC, and FHD was input in model 4 to predict prediabetes. ROC curve analysis was finally conducted to evaluate the predictive ability of the model for prediabetes $(\mathrm{AUC}=0.702$; Figure 2). AIC, NRI, and IDI were used to compare the prediction models. They exhibited better predictive values of FPG, $\mathrm{HbAlc}$, WC, and FHD for prediabetes (Table 3). This is the first study to construct a multivariate prediction model, which included demographic characteristics, anthropometric measurements, and metabolic profiles for Chinese adults.

The functions of islets gradually decline with age, including weakened insulin action, leading to lipid and glucose metabolism disorder. Prediabetes incidences are correlated with age [27]. Gender has also been reported to be correlated with prediabetes incidences. For instance, the prevalence of prediabetes was significantly higher in males than in females [28]. However, it was reported that gender and age were not correlated with prediabetes in another study [29]. This is in line with our study, potentially due to the sample size and differences in the districts of participants. This warrants further investigations.

This work has some limitations. First, the study population is from a single community in southern China, with limited representation. Therefore, the true effect of this model should be further investigated and verified in different populations. Second, correlations stratified by age and gender were unexplored because of sample size limitations. Third, although many people were lost to follow-up, no significant statistical differences were noted in basic characteristics for the 243 lost and 551 follow-up participants.
Therefore, the 551 followed up participants may validly represent the entire study cohort. Despite its limitations, the model can still be used, given that few models have been developed to predict prediabetes in the Chinese population.

\section{Conclusions}

FPG, HbAlc, WC, and FHD are independently correlated with the increased risk of prediabetes. A combination of these predictors enhances the predictive accuracy for prediabetes. We developed a multivariate prognostic model to predict the risk of prediabetes development. This may help in identifying high-risk populations and to develop preventive strategies.

\section{Data Availability}

The data used to support the findings of this study are available from the corresponding author upon request.

\section{Ethical Approval}

The authors certify that they complied with the ethical guidelines for authorship and publishing. This study was approved by the ethics committee of Sir Run Run Shaw Hospital.

\section{Consent}

All the patients signed an informed consent form to participate.

\section{Conflicts of Interest}

The authors declare that they have no conflicts of interest.

\section{Acknowledgments}

This work was supported by the National Key Technology R\&D Program of China (Grant nos. 2009BAI80B00 and 2012BAI02B03).

\section{Supplementary Materials}

Table S1: univariate Cox proportional-hazards regression models for predicting prediabetes. (Supplementary Materials) 


\section{References}

[1] D. M. Nathan, M. B. Davidson, R. A. DeFronzo et al., "Impaired fasting glucose and impaired glucose tolerance: implications for care," Diabetes Care, vol. 30, no. 3, pp. 753-759, 2007.

[2] A. G. Tabák, C. Herder, W. Rathmann, E. J. Brunner, and M. Kivimäki, "Prediabetes: a high-risk state for diabetes development," The Lancet, vol. 379, no. 9833, pp. 2279-2290, 2012.

[3] Y. Huang, X. Cai, W. Mai, M. Li, and Y. Hu, "Association between prediabetes and risk of cardiovascular disease and all cause mortality: systematic review and meta-analysis," BMJ, vol. 355, Article ID i5953, 2016.

[4] P. K. Crane, R. Walker, R. A. Hubbard et al., "Glucose levels and risk of dementia," The New England Journal of Medicine, vol. 369, no. 6, pp. 540-548, 2013.

[5] N. H. Cho, J. E. Shaw, S. Karuranga et al., "IDF Diabetes Atlas: global estimates of diabetes prevalence for 2017 and projections for 2045," Diabetes Research and Clinical Practice, vol. 138, pp. 271-281, 2018.

[6] Y. Li, D. Teng, X. Shi, G. Qin, Y. Qin, and H. Quan, "Prevalence of diabetes recorded in mainland China using 2018 diagnostic criteria from the American Diabetes Association: National cross sectional study," BMJ, vol. 369, Article ID m997, 2020.

[7] X. R. Pan, G. W. Li, Y. H. Hu et al., "Effects of diet and exercise in preventing NIDDM in people with impaired glucose tolerance: the Da Qing IGT and diabetes study," Diabetes Care, vol. 20, no. 4, pp. 537-544, 1997.

[8] K. P. Vatcheva, S. P. Fisher-Hoch, B. M. Reininger, and J. B. McCormick, "Sex and age differences in prevalence and risk factors for prediabetes in Mexican-Americans," Diabetes Research and Clinical Practice, vol. 159, Article ID 107950, 2020.

[9] K. K. Aldossari, A. Aldiab, J. M. Al-Zahrani et al., "Prevalence of prediabetes, diabetes, and its associated risk factors among males in Saudi Arabia: a population-based survey," Journal of Diabetes Research, vol. 2018, Article ID 2194604, 2018.

[10] V. Tsimihodimos, C. Gonzalez-Villalpando, J. B. Meigs, and E. Ferrannini, "Hypertension and diabetes mellitus coprediction and time trajectories," Hypertension, vol. 71, no. 3, pp. 422-428, 2018.

[11] J. Wen, A. Wang, G. Liu et al., "Elevated triglyceride-glucose (TyG) index predicts incidence of prediabetes: a prospective cohort study in China," Lipids in Health and Disease, vol. 19, pp. 1-10, 2020.

[12] J. Wu, L. Gong, Q. Li et al., "A novel visceral adiposity index for prediction of type 2 diabetes and pre-diabetes in Chinese adults: a 5-year prospective study," Scientific Reports, vol. 7, pp. 1-9, 2017.

[13] S. M. Ruchat, T. Rankinen, S. J. Weisnagel et al., "Improvements in glucose homeostasis in response to regular exercise are influenced by the PPARG Pro12Ala variant: results from the HERITAGE family study," Diabetologia, vol. 53, no. 4, pp. 679-689, 2010.

[14] X. M. Shen, Y. Q. Huang, X. Y. Zhang, X. Q. Tong, P. F. Zheng, and L. Shu, "Association between dietary patterns and prediabetes risk in a middle-aged Chinese population," Nutrition Journal, vol. 19, no. 1, p. 77, 2020.

[15] D. Tricò, A. Galderisi, A. Mari, N. Santoro, and S. Caprio, "One-hour post-load plasma glucose predicts progression to prediabetes in a multi-ethnic cohort of obese youths,"
Diabetes, Obesity and Metabolism, vol. 21, no. 5, pp. 1191-1198, 2019.

[16] H. Wang, X. Zheng, Z. H. Bai et al., "A retrospective population study to develop a predictive model of prediabetes and incident type 2 diabetes mellitus from a hospital database in Japan between 2004 and 2015," Medical Science Monitor, vol. 26, Article ID e920880, 2020.

[17] I. I. Fujiati, H. A. Damanik, A. Bachtiar, A. A. Nurdin, and P. Ward, "Development and validation of prediabetes risk score for predicting prediabetes among Indonesian adults in primary care: cross-sectional diagnostic study," Interventional Medicine and Applied Science, vol. 9, no. 2, pp. 76-85, 2017.

[18] Y. Xueyao, Z. Saifei, Y. Dan et al., "Circulating fractalkine levels predict the development of the metabolic syndrome," The Internet Journal of Endocrinology, vol. 2014, Article ID 715148, 2014.

[19] A. Tchernof and J. P. Després, "Pathophysiology of human visceral obesity: an update," Physiological Reviews, vol. 93, no. 1, pp. 359-404, 2013.

[20] S. E. Kahn, R. L. Hull, and K. M. Utzschneider, "Mechanisms linking obesity to insulin resistance and type 2 diabetes," Nature, vol. 444, no. 7121, pp. 840-846, 2006.

[21] R. Wagner, B. Thorand, M. A. Osterhoff, G. Müller, A. Böhm, and C. Meisinger, "Family history of diabetes is associated with higher risk for prediabetes: a multicentre analysis from the German center for diabetes research," Diabetologia, vol. 56, no. 10, pp. 2176-2180, 2013.

[22] M. Rodríguez-Moran, F. Guerrero-Romero, C. AradillasGarcía et al., "Obesity and family history of diabetes as risk factors of impaired fasting glucose: implications for the early detection of prediabetes," Pediatric Diabetes, vol. 11, no. 5, pp. 331-336, 2010.

[23] G. P. Watt, K. P. Vatcheva, D. M. Griffith et al., "The precarious health of young Mexican American men in South Texas, cameron county hispanic cohort, 2004-2015," Preventing Chronic Disease, vol. 13, p. E113, 2016.

[24] M. Janghorbani and M. Amini, "Normal fasting plasma glucose and risk of prediabetes and type 2 diabetes: the Isfahan diabetes prevention study," The Review of Diabetic Studies, vol. 8, no. 4, pp. 490-498, 2011.

[25] F. Taheri, B. Bijari, T. Kazemi, K. Namakin, M. Zardast, and T. Chahkandi, "Prevalence of high normal FBS and prediabetes among adolescents in Birjand, East of Iran, 2012," Journal of Education and Health Promotion, vol. 4, p. 68, 2015.

[26] L. Luo, X. Li, and Z. Gao, "The value of waist circumference, waist height ratio and visceral adiposity index in predicting new prediabetes in women over 40 years old in Dalian," Chinese Journal of Diabetes, vol. 28, pp. 17-22, 2020.

[27] T. H. T. Chiu, H. Y. Huang, Y. F. Chiu et al., "Taiwanese vegetarians and omnivores: dietary composition, prevalence of diabetes and IFG," PLoS One, vol. 9, no. 2, Article ID e88547, 2014.

[28] I. S. K. Krishnadath, L. M. N. Nahar-van Venrooij, V. W. V. Jaddoe, and J. R. Toelsie, "Ethnic differences in prediabetes and diabetes in the Suriname health study," BMJ Open Diabetes Research \& Care, vol. 4, no. 1, Article ID e000186, 2016.

[29] I. O. Okwechime, S. Roberson, and A. Odoi, "Prevalence and predictors of pre-diabetes and diabetes among adults 18 years or older in Florida: a multinomial logistic modeling approach," PLoS One, vol. 10, no. 12, Article ID e0145781, 2015. 